\title{
Targeted inhibition of mitochondrial Hsp90 induces mitochondrial elongation in Hep3B hepatocellular carcinoma cells undergoing apoptosis by increasing the ROS level
}

\author{
SEUNG HEE YOO ${ }^{1}$, HYE YOUNG KIM ${ }^{1}$, JEE HYUN RHO ${ }^{1}$, SEON-YONG JEONG ${ }^{2}$, \\ JEANHO YUN $^{3}$, IL YUN $^{4}$, HWAN TAE PARK $^{5}$ and YOUNG HYUN YOO ${ }^{1}$ \\ ${ }^{1}$ Department of Anatomy and Cell Biology and Mitochondria Hub Regulation Center, College of Medicine, \\ Dong-A University, Busan; ${ }^{2}$ Department of Medical Genetics, School of Medicine, Ajou University, Suwon; \\ ${ }^{3}$ Department of Biochemistry, Dong-A University College of Medicine, Busan; ${ }^{4}$ Department of Dental Pharmacology \\ and Biophysics, School of Dentistry and Research Institute for Oral Biotechnology, Yangsan Campus of Pusan \\ National University, Yangsan; ${ }^{5}$ Department of Physiology and Mitochondria Hub Regulation Center, \\ Dong-A University College of Medicine, Busan, Republic of Korea
}

Received July 2, 2015; Accepted August 12, 2015

DOI: 10.3892/ijo.2015.3150

\begin{abstract}
Previous studies reported that a Gamitrinib variant containing triphenylphosphonium (G-TPP) binds to mitochondrial Hsp90 and rapidly inhibits its activity to induce apoptosis. We investigated the mechanisms underlying the antitumor activity of G-TPP in Hep3B hepatocellular carcinoma cells. Contrary to our predictions, we observed mitochondrial elongation in the G-TPP-treated Hep3B cells undergoing apoptosis. We found that the G-TPP-induced mitochondrial elongation in Hep3B cells was caused by a decrease in the mitochondrial fission-regulating protein Drp1 rather than by changes in the mitochondrial fusion machinery proteins Mfn1 and Opa1. Furthermore, G-TPP induced G2-M phase cell cycle arrest by reducing the interaction between CDK1 and cyclin B1. Additionally, reactive oxygen species (ROS) played a pivotal role in G-TPP-induced cell death and mitochondrial elongation in Hep3B cells, and these processes are mediated by the reduced association of CDK1 with cyclin B1 and the suppressed phosphorylation of Drp1 (Ser616). Thus, G-TPP induces cell death and causes Drp1-mediated mitochondrial elongation in Hep3B cells by increasing the ROS level.
\end{abstract}

Correspondence to: Dr Hwan Tae Park, Department of Physiology, Dong-A University College of Medicine, 3-1 Dongdaeshin-dong, Seo-gu, Busan 602-714, Republic of Korea

E-mail:phwantae@dau.ac.kr

Dr Young Hyun Yoo, Department of Anatomy and Cell Biology, Dong-A University College of Medicine, 3-1 Dongdaeshin-dong, Seo-gu, Busan 602-714, Republic of Korea

E-mail: yhyoo@dau.ac.kr

Key words: G-TPP, mitochondrial dynamics, Drp1, reactive oxygen species, Hep3B cells

\section{Introduction}

Mitochondria are highly dynamic organelles that continuously elongate and divide to form a network throughout the cell. The shape, location and function of mitochondria are defined by an equilibrium between opposing fusion and fission events (1). Mitochondrial dynamics are crucial to homeostasis and cellular energy production $(2,3)$. Mitochondrial fusion and fission are precisely controlled by various mitochondria-shaping proteins (4-6). In mammalian cells, three large GTPases, mitofusin 1 (Mfn1), mitofusin 2 (Mfn2) and optic atrophy 1 (Opal), are essential for mitochondrial fusion. Mfns are integrated into the outer mitochondrial membrane (OMM) and form homo- and hetero-oligomers, which promote the tethering and fusion of OMMs from two different mitochondria (1). Opa1 localizes in complexes at the inner mitochondrial membrane (IMM) and drives fusion on the IMM (7). A GTPase cytosolic dynamin-related protein 1 (Drp1) mediates mitochondrial fission in mammalian cells. To constrict and cut mitochondria during mitochondrial fission, Drp1, which is located in the cytosol, needs to be activated and assembled onto mitochondria (8). Fisson 1 (Fis1), which is located on the OMM, has been suggested to be a Drp1 receptor and required for mitochondrial fission $(1,9)$. However, its action mechanism remains highly controversial (10).

Various stimuli such as anticancer agents, hypoxia or radiation can induce mitochondrial dysfunction. Damaged mitochondria may be repaired or removed. Mitochondrial fission is a crucial mechanism for removing dysfunctional mitochondria via mitophagy (11). Crosstalk between apoptosis, mitophagy and mitochondrial dynamics seems to be critical to the overall fate of cells, i.e. death or survival (12). Previous studies reported that apoptosis-inducing agents generally induce mitochondrial fragmentation (13-16).

Previous studies have reported that a Gamitrinib variant containing triphenylphosphonium (G-TPP) readily accumulated in the mitochondria of normal or tumor cells and 
inhibited the tumor necrosis factor receptor-associated protein 1 (TRAP1) and mitochondrial heat shock protein 90 (Hsp90) inside the mitochondria. G-TPP reduced the IMM potential and caused the discharge of apoptogenic proteins into the cytosol by activating cyclophilin D-dependent mitochondrial permeability in various cancer cells, which resulted in apoptosis $(17,18)$. Additionally, G-TPP binds to mitochondrial Hsp90, which causes apoptosis by activating cyclophilin D-dependent mitochondrial permeability transition in tumor cells $(18,19)$.

We observed that G-TPP induces cell death and causes Drp1-mediated mitochondrial elongation in Hep3B cells by increasing the reactive oxygen species (ROS) level.

\section{Materials and methods}

Cell culture. Hep3B cells obtained from the American Type Culture Collection were maintained in Dulbecco's modified Eagle's medium (DMEM) containing 10\% heat-inactivated fetal bovine serum (FBS) and $1 \%$ (v/v) penicillin-streptomycin (PS) at $37^{\circ} \mathrm{C}$ in a $5 \% \mathrm{CO}_{2}$ humid atmosphere. After $48 \mathrm{~h}$ of culture, the medium was removed from the Hep3B cells, which were then washed with PBS and incubated in the same fresh medium.

The establishment of parkin-YFP-overexpressing Hep3B cells. The parkin-YFP plasmid was provided by Dr J. Chung (Seoul National University, Seoul, Korea). To establish cell lines that stably expressed parkin-YFP, Hep3B cells were seeded into 6 -well plates $\left(2 \times 10^{5}\right.$ cells/well) for $24 \mathrm{~h}$ prior to transfection. The cells were transfected with $2 \mu \mathrm{g}$ of parkin-YFP plasmid using Lipofectamine 2000 according to the manufacturer's instructions. Stably transfected Hep3B/parkin-YFP cells were selected by incubating the cells in medium containing $500 \mu \mathrm{g} / \mathrm{ml}$ of neomycin sulfate (G418) for 2 weeks. The overexpression of parkin-YFP was confirmed by observing cells under Zeiss LSM 700 laser-scanning confocal microscope (Goettingen, Germany).

Transfection of silencing RNA (siRNA). Hep3B cells growing into 6 -well plates $\left(0.8 \times 10^{5}\right.$ cells/well $)$ were transfected with siRNA. siRNAs against the human Mfn1, Opa1, Drp1 transcripts were purchased from Dharmacon (ON-TARGETplus SMARTpool siRNA) and used at a concentration of $10 \mathrm{nM}$. As a negative control, the same nucleotide was scrambled to generate a non-targeting siRNA. The siRNAs were transfected into Hep3B cells using Lipofectamine ${ }^{\circledast}$ RNAiMAX per the manufacturer's instructions.

Reagents. The reagents were obtained from commercial sources: DMEM and FBS were obtained from Gibco-BRL (Gaithersburg, MD, USA); rabbit polyclonal antibodies to human Mfn1 (sc-50330), Tom20 (sc-11415) and cyclin B1 (sc-752), and mouse monoclonal antibody to human CDK1 (sc-54) were obtained from Santa Cruz Biotechnology (Santa Cruz, CA, USA); mouse monoclonal antibodies to human Drp1 (61112) and Opa1 (612606) were obtained from BD Transduction Laboratories (Lexington, KY, USA); rabbit polyclonal antibodies against human caspase-3 (\#9662), caspase-7 (\#9492) and p-Drp1 (Ser616) (\#3455) as well as
HRP-conjugated goat anti-rabbit and horse anti-mouse IgG antibodies were obtained from Cell Signaling Technologies (Danvers, MA, USA), as was also the RIPA buffer. Texas Red-conjugated goat anti-rabbit IgG antibody was obtained from Vector (Burlingame, CA, USA); mouse monoclonal antibodies against human $\beta$-actin (A5441) and $\alpha$-tubulin (T5168) as well as Hoechst 33342, protein-A agarose, $N$-acetyl-Lcysteine (NAC), propidium iodide (PI), carbonyl cyanide m-chlorophenylhydrazone (CCCP) and the Annexin V-FITC apoptosis detection kit were obtained from Sigma-Aldrich (Irvine, CA, USA). G418 was purchased from Duchefa (Haarlem, The Netherlands). 5,5',6,6'-Tetrachloro-1,1',3,3'tetraethyl benzimidazole carbocyanine iodide (JC-1) and MitoSOX $^{\text {TM }}$ Red reagent were purchased from Molecular Probes (Eugene, OR, USA). Lipofectamine ${ }^{\circledast}$ RNAiMAX reagent and Lipofectamine 2000 were obtained from Invitrogen (Carlsbad, CA, USA). The SuperSignal West Pico enhanced chemiluminescence western blotting detection reagent was purchased from Pierce (Rockford, IL, USA) and RNase A was purchased from Biosesang (Biosesang, Inc., Korea).

Chemical synthesis of G-TPP. G-TTP was synthesized by LegoChem Biosciences Inc. (Daejeon, Korea) as previously described (18).

Cell viability assay. The cell viability was assessed using the Vi-Cell cell counter (Beckman Coulter, Miami, FL, USA) to perform an automated trypan blue exclusion assay.

Western blot analysis. The cells were washed twice with ice-cold PBS, resuspended in RIPA buffer and incubated at $4^{\circ} \mathrm{C}$ for $30 \mathrm{~min}$. The lysates were centrifuged at $14,000 \mathrm{rpm}$ for $20 \mathrm{~min}$ at $4^{\circ} \mathrm{C}$. The protein concentrations of the cell lysates were determined with the Bradford protein assay reagent (Bio-Rad), and $30 \mu \mathrm{g}$ of protein was loaded onto $7.5-15 \%$ SDS/PAGE gels. The proteins were transferred to nitrocellulose membranes (Amersham Pharmacia Biotech, Piscataway, NJ, USA). The bands were visualized by incubation with 1:1,000 dilutions of primary antibody overnight at $4^{\circ} \mathrm{C}$, followed by incubation with 1:2,000 dilutions of secondary antibody at room temperature for $1 \mathrm{~h}$. The antibody signal was developed using the SuperSignal West Pico-enhanced chemiluminescence substrate and detected with a LAS-4000PLUS (Fuji Photo Film, Tokyo, Japan).

Subcellular fractionation. The cells $\left(3 \times 10^{7}\right.$ cells) were washed in ice-cold Tris-based $\mathrm{Mg}^{2+} / \mathrm{Ca}^{2+}$-free buffer $(135 \mathrm{mM} \mathrm{NaCl}$, $5 \mathrm{mM} \mathrm{KCl}$ and $25 \mathrm{mM}$ Tris- $\mathrm{HCl} \mathrm{pH}$ 7.4). The mitochondrial and cytosolic fractions were isolated using the Mitochondrial Fractionation kit (Active Motif, Carlsbad, CA, USA). The cells were resuspended in cytosolic buffer and incubated on ice for $15 \mathrm{~min}$. The cells were then homogenized on ice with a homogenizer operated at 60 strokes. The lysates were centrifuged at $3,000 \mathrm{rpm}$ and $4^{\circ} \mathrm{C}$ for $15 \mathrm{~min}$. The supernatant contained the cytosol, including the mitochondria. The supernatant was transferred to a microcentrifuge tube and centrifuged at $13,000 \mathrm{rpm}$ and $4^{\circ} \mathrm{C}$ for $30 \mathrm{~min}$ to pellet the mitochondria. The mitochondrial pellets were lysed with mitochondrial buffer on ice for $15 \mathrm{~min}$ and then centrifuged at 13,000 rpm for $30 \mathrm{~min}$ at $4^{\circ} \mathrm{C}$. 
Co-immunoprecipitation (Co-IP). After being incubated with antibodies, the cell extracts were precipitated with protein A-Sepharose beads for $3 \mathrm{~h}$ and washed 3 times with an extraction buffer prior to boiling them in the SDS sample buffer. The immunoprecipitated proteins were separated using SDS-PAGE, and a western blot analysis was performed as described above.

Observation and quantification of mitochondrial morphology using confocal microscopy. The cells were cultured on coverslips and fixed with $4 \%$ paraformaldehyde for $1 \mathrm{~h}$. The cells were then permeabilized with $0.2 \%$ Triton $\mathrm{X}-100$ for $15 \mathrm{~min}$ and incubated with Tom 20 antibody for $1 \mathrm{~h}$ at room temperature (RT). They were washed 3 times with PBS for 5 min each, incubated with a Texas Red-conjugated secondary antibody for $1 \mathrm{~h}$ at room temperature and counterstained with Hoechst 33342. A Zeiss LSM 700 laser-scanning confocal microscope at a magnification of $x 40$ ( 0.55 numerical aperture) was used to obtain and analyze the fluorescent images. The cells were divided into the following 3 groups based on their mitochondrial morphology: fragmented, cells that primarily contained mitochondria shorter than $2 \mu \mathrm{m}$; tubular and donuts, cells that primarily contained mitochondria between $\sim 2$ and $5 \mu \mathrm{m}$ long; and elongated, cells that primarily contained mitochondria $>5 \mu \mathrm{m}$. Three independent experiments were conducted, and 100 cells were scored per experiment.

Quantification of DNA hypoploidy and cell cycle phase analysis by flow cytometry. Ice-cold $95 \%$ ethanol containing $0.5 \%$ Tween-20 was added to the cell suspension to a final concentration of $70 \%$ ethanol. The fixed cells were pelleted and washed in $1 \%$ BSA-PBS solution. They were re-suspended in $1 \mathrm{ml}$ of PBS containing 11 Kunitz U/ml RNase A, incubated at $37^{\circ} \mathrm{C}$ for $1 \mathrm{~h}$, washed once with BSA-PBS, re-suspended in PI solution $(10 \mu \mathrm{g} / \mathrm{ml})$, and incubated in the dark at $4^{\circ} \mathrm{C}$ for 30 min. The cells were then washed with PBS, and the DNA content was measured on an Epics XL (Beckman Coulter). The data were analyzed using the MultiCycle software, which allowed the simultaneous estimation of cell cycle parameters and apoptosis.

Mitochondrial membrane potential (MMP) assay. To measure the MMP, the cells were trypsinized, collected, stained with JC-1 and subjected to flow cytometry using an Epics XL flow cytometer (Beckman Coulter). The data were acquired and analyzed using the EXPO32 ADC XL 4 color software program.

Flow cytometric analysis of Annexin V-FITC. The cells were trypsinized, collected and stained with the Annexin V-FITC apoptosis detection kit according to the manufacturer's instructions. After centrifugation, cells were resuspended in PBS and analyzed with Epics XL.

Measurement of ROS levels. The cells were trypsinized, collected and stained with $5 \mu \mathrm{M}$ MitoSox for $30 \mathrm{~min}$ at $37^{\circ} \mathrm{C}$. After centrifugation, the cells were resuspended in PBS and analyzed with Epics XL.

Statistical analysis. At least three independent experiments were carried out in vitro. The results are expressed as the means \pm SD from three experiments. The significance of differences was determined using the paired Kruskal-Wallis non-parametric test. A p-value $<0.05$ was considered significant.

\section{Results}

$G$-TPP induces cell death and mitochondrial elongation in Hep3B cells. Treating Hep3B cells with 1-80 $\mu \mathrm{M}$ G-TPP for $48 \mathrm{~h}$ significantly reduced their viability in a dose-dependent manner (Fig. 1A). Because the viability of Hep3B cells treated with $40 \mu \mathrm{M}$ G-TTP for $48 \mathrm{~h}$ was $\sim 50 \%$, this concentration was used for further studies. G-TPP treatment reduced the viability of Hep3B cells in a time-dependent manner (Fig. 1B). To examine whether the reduced viability of G-TTP-treated Hep3B cells was due to apoptotic cell death, we performed various apoptosis assays. The caspase cleavage and mitochondrial membrane potential assays indicated that G-TPP at least partly induced Hep3B cell death via apoptosis (Fig. 1C and D). Notably, G-TPP treatment significantly increased the population of Hep3B cells that contained elongated mitochondria (Fig. 1E and F).

Drpl is involved in G-TPP-induced mitochondrial elongation. We examined whether the G-TPP-induced mitochondrial elongation was mediated by alterations in the mitochondrial fusion-regulating proteins Mfn1 and Opa1. The western blot assay showed that G-TPP did not increase the expression levels of Mfn1 and Opa1 (Fig. 2A). We then examined the effect of Mfn1 or Opa1 depletion on G-TPP-induced cell death. A viability assay showed that neither siMfn1 nor siOpal significantly alter G-TPP-induced cell death (Fig. 2B). As predicted, both siMfn1 and siOpa1 significantly increased the population of G-TPP-untreated Hep3B cells that contained fragmented mitochondria. However, treatment with G-TPP significantly increased the populations of both Mfn1- and Opa1-depleted Hep3B cells that contained elongated mitochondria (Fig. 2C and D). These data indicate that G-TPP-induced mitochondrial elongation is not mediated by an increase in the mitochondrial fusion proteins Mfn1 and Opa1. We next examined whether the G-TPP-induced mitochondrial elongation was mediated by alterations in the fission-regulating protein Drp1. The western blot assay showed that treatment with G-TPP markedly decreased the Drp1 level in Hep3B cells, particularly in the mitochondria (Fig. 3A). We next examined the effect of Drp1 depletion on G-TPP-induced cell death. siDrpl did not significantly alter the viability of Hep3B cells treated with G-TPP (Fig. 3B). However, siDrp1 significantly increased the populations of both G-TPP-treated and untreated Hep3B cells that contained elongated mitochondria compared with scrambled siRNA (Fig. 3C). Confocal microscopy demonstrated that mitochondria are more aggregated in Hep3B cells treated with G-TPP plus siDrp1 than cells treated with G-TPP plus scrambled siRNA (Fig. 3D). These data indicated that G-TPP-induced mitochondrial elongation was caused by Drp1 reduction.

G-TPP suppresses the translocation of Drpl into mitochondria in parkin-overexpressing Hep3B cells. We investigated whether G-TPP suppressed the translocation of Drp1 into mitochondria in parkin-overexpressing Hep3B cells. We 
A

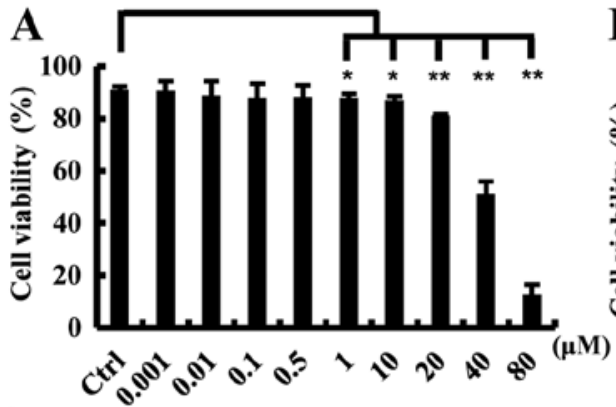

D
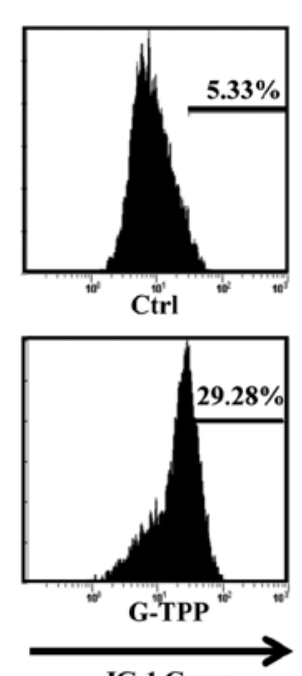

JC-1 Green

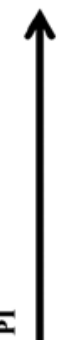

$\bar{a}$
B
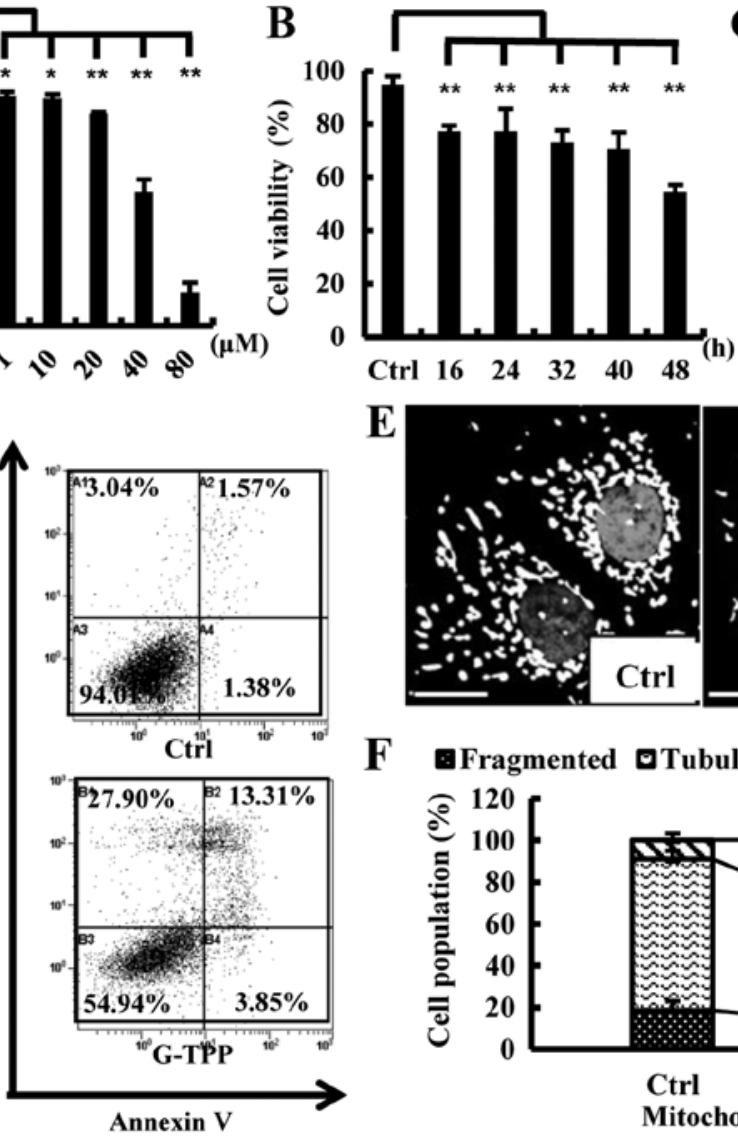

$\mathbf{E}$

$\mathrm{C}$
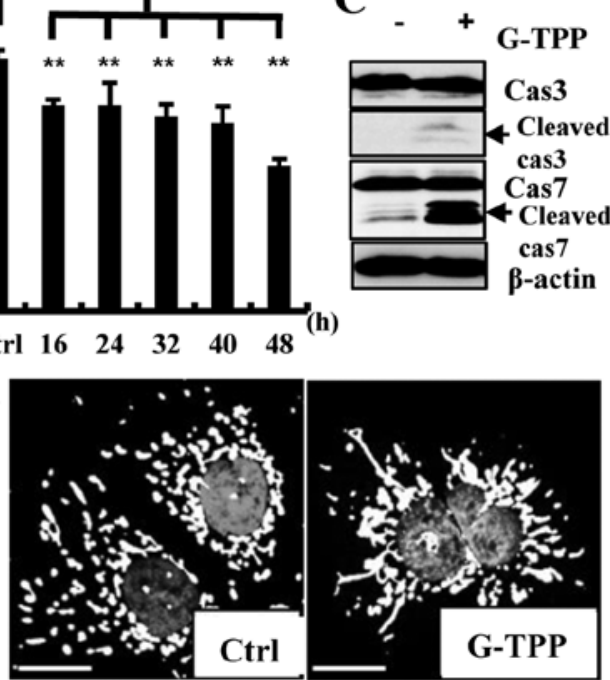

F

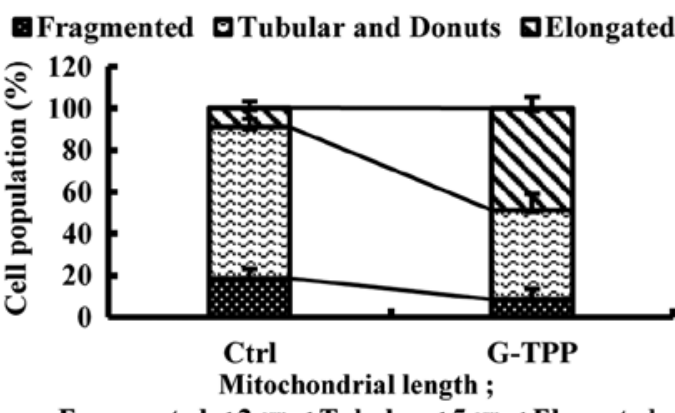

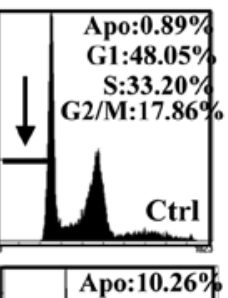

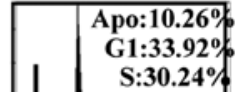

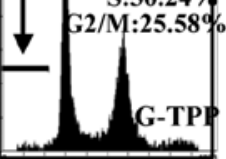

Fragmented $<2 \mu \mathrm{m}<$ Tubular $<5 \mu \mathrm{m}<$ Elongated

Figure 1. G-TPP induced cell death and mitochondrial elongation in Hep3B cells. Ctrl; control, G-TPP; Gamitrinib variant containing triphenylphosphonium, Cas3; caspase-3, Cas7; caspase-7. (A) Viability after treatment with 0.001-80 $\mu \mathrm{M}$ G-TPP for $48 \mathrm{~h}$. Treatment with $1-80 \mu \mathrm{M}$ G-TPP significantly reduced the viability compared with the control. ${ }^{*} \mathrm{p}<0.05,{ }^{* *} \mathrm{p}<0.01$. (B) Viability after treatment with $40 \mu \mathrm{M}$ G-TPP for $16-48$ h. Treatment with G-TPP reduced viability compared with the control. ${ }^{* *} \mathrm{p}<0.01$. (C) Western blot analyses showing the production of cleaved caspase- 3 and -7 in response to $\mathrm{G}-\mathrm{TPP}$ treatment. $\beta$-actin served as a loading control (left panel). Representative histograms showing that G-TPP induced the accumulation of subdiploid apoptotic Hep3B cells. Apo, the percentage of the population undergoing apoptosis (arrows in right panel). (D) Flow cytometry using JC-1 showing that G-TPP reduced the MMP of Hep3B cells (left panel). Flow cytometry using Annexin V-FITC/PI double staining showing that G-TPP increased the population of Annexin V-positive cells (right panel). (E) Confocal microscopy showing that G-TPP induced mitochondrial elongation. To visualize the mitochondria and nuclei, cells were stained with Tom 20 (white) and Hoechst 33342 (gray). Scale bar, $10 \mu \mathrm{m}$. (F) Quantification of the percentage of cells containing fragmented (dotted), tubular and donut-shaped (short line) or elongated (striped) mitochondria. G-TPP treatment significantly increased the population of elongated cells $(\mathrm{p}<0.01)$. The graphs are averages of three independent experiments, with 100 cells scored per experiment.

observed that parkin translocated into the mitochondria of parkin-overexpressing Hep3B cells, irrespective of G-TPP treatment. Additionally, parkin translocated into the fragmented mitochondria of parkin-overexpressing Hep3B cells treated with the representative mitophagy inducer CCCP (Fig. 4A). A western blot assay demonstrated that G-TPP decreased the expression level of Drp1 not only in Hep3B cells but also in parkin-overexpressing Hep3B cells. G-TPP also inhibited the translocation of Drp1 into mitochondria in Hep3B cells. Importantly, CCCP increased the expression level of Drp1 and augmented the mitochondrial translocation of Drp1 in the parkin-overexpressing Hep3B cells. G-TPP inhibited the translocation of Drp1 into mitochondria in the parkin-overexpressing Hep3B cells (Fig. 4B). The population of cells that contained elongated or fragmented mitochondria did not differ between control Hep3B and parkin-overexpressing Hep3B cells treated with G-TPP (Fig. 4C). Furthermore, the overexpression of parkin did not affect the level of G-TPP-induced cell death (Fig. 4D). These data indicate that G-TPP inhibited the mitochondrial translocation of Drp1 even in the parkin-overexpressing Hep3B cells, which may impair parkin-mediated mitophagy.

$G$-TPP reduces the interaction of $C D K 1$ with cyclin $B 1$ and the activating phosphorylation of Drpl (Ser616). Flow cytometry revealed an increase in the percentage of G2-M phase cells and a concomitant decrease in the percentage of the G1 phase cells (Fig. 1C), which indicated that G-TPP induced G2-M phase cell cycle arrest in Hep3B cells. Therefore, we examined the association between mitochondrial elongation and cell cycle progression in G-TPP-treated Hep3B cells. We examined the level of CDK1 and cyclin B1 with a western blotting, which showed that G-TPP markedly decreased the expression level of CDK1. G-TPP also markedly reduced the activating phosphorylation of Drp1 (Ser616) (Fig. 5A). Because the CDK1-cyclin B1 complex is known to be associated with the activation of Drp1 via the phosphorylation of Drp1 at Ser616 (pDrp1-Ser616) (20), we examined the effect of G-TPP on the formation of the CDK1-cyclin B1 complex. The co-immunoprecipitation results indicated that G-TPP reduced 
A
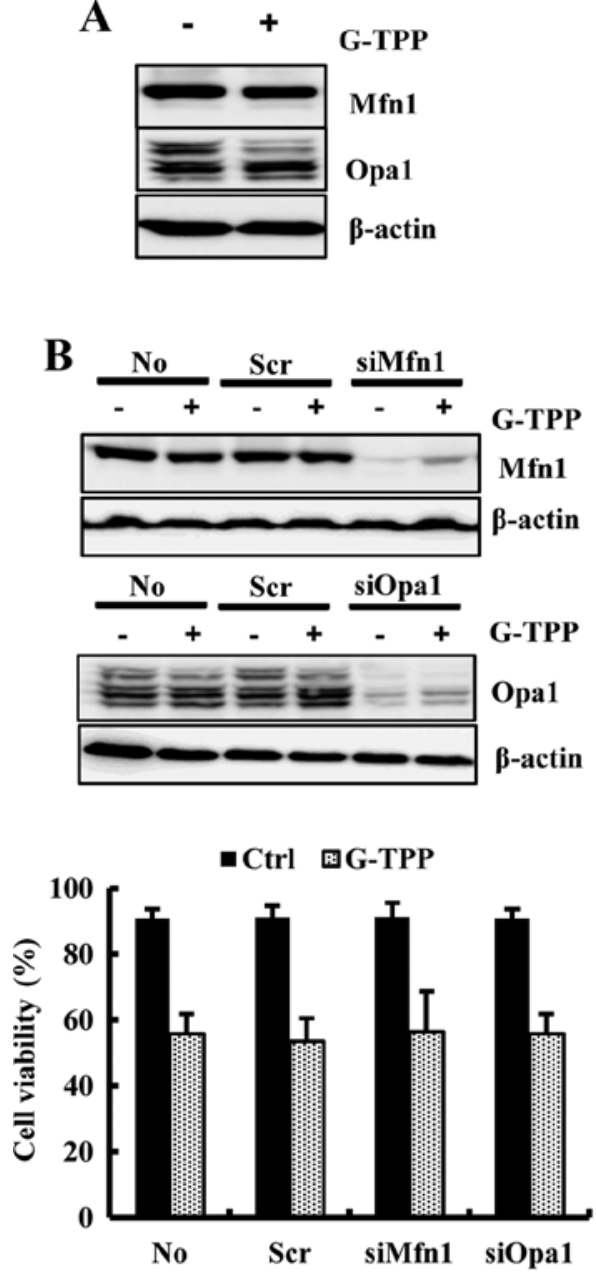

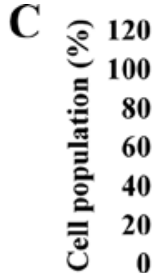

D

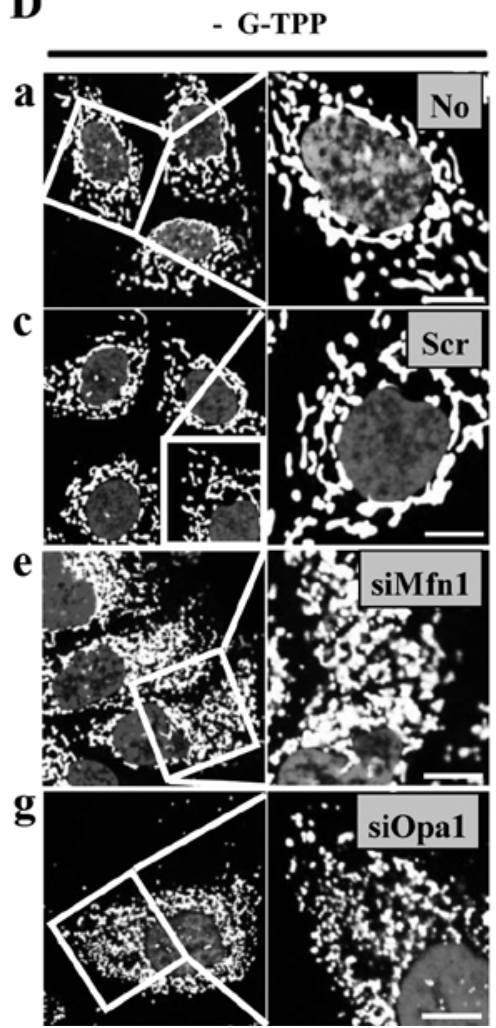

G Fragmented QTubular and Donuts DElongated

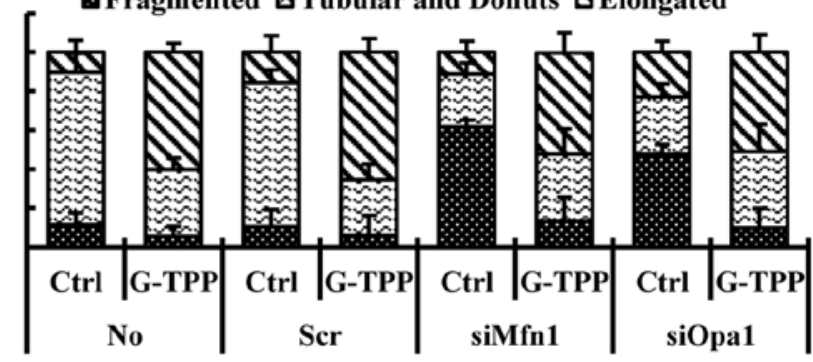

Mitochondrial length ; Fragmented $<2 \mu \mathrm{m}<$ Tubular $<5 \mu \mathrm{m}<$ Elongated

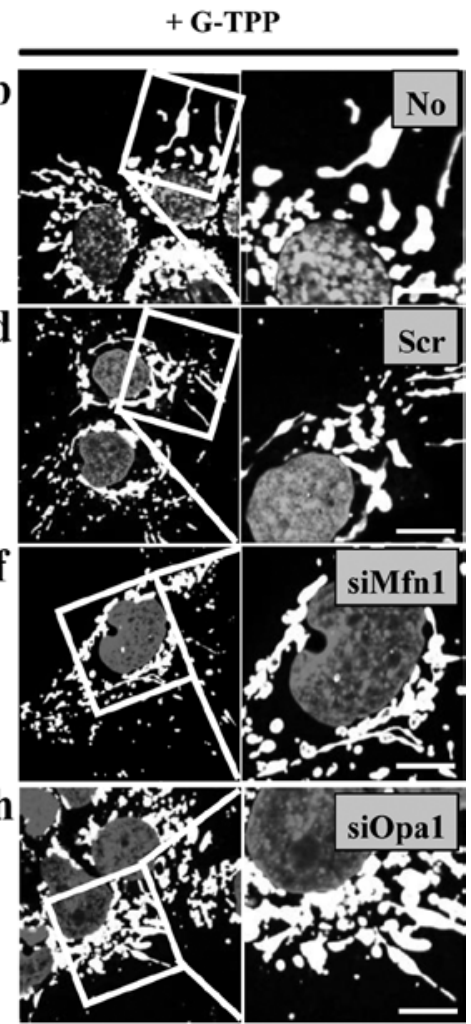

Figure 2. Depletion of mitochondrial fusion factors using siRNA affected neither the cellular viability nor mitochondrial length in G-TPP-treated Hep3B cells. Cells were transfected with scrambled siRNA (Src) or siRNA targeting Mfn1 or Opa1. After 16 h, the cells were treated with $40 \mu \mathrm{M}$ G-TPP for $48 \mathrm{~h}$ (A) Western blot analyses showing that G-TPP did not increase the expression level of Mfn1 and Opa1. (B) Western blot analyses showing that Mfn1 and Opa1 were efficiently depleted in Hep3B cells by their specific siRNA. $\beta$-actin served as a loading control (upper panel). Viability assay showing siRNA against Mfn1 or Opa1 did not affect the cell viability (lower panel). (C and D) Neither siMfn1 nor siOpa1 significantly altered G-TPP-induced mitochondrial elongation in Hep3B cells. (C) Quantification of the percentage of cells containing fragmented (dotted), tubular and donut-shaped (short line) or elongated (striped) mitochondria. The graphs are averages of three independent experiments, with 100 cells scored per experiment. (D) Confocal microscopy showing mitochondrial morphology. To visualize the mitochondria and nuclei, cells were stained with Tom 20 (white) and Hoechst 33342 (gray). Scale bar, $10 \mu \mathrm{m}$. See the legend in Fig. 1 for other definitions.

the level of CDK1-cyclin B1 complex formation (Fig. 5B). These data indicated that G-TPP reduced the interaction between CDK1 and cyclin B1 and thereby inhibited its Drp1 activation and mitochondrial localization, which induced mitochondrial elongation.

ROS mediates G-TPP-induced cell death and mitochondrial elongation. Next, we examined the involvement of ROS in G-TPP-induced cell death and mitochondrial elongation in Hep3B cells. We observed that G-TPP increased the ROS level in Hep3B cells, and the ROS scavenger NAC remarkably inhibited the level of ROS in the G-TPP-treated Hep3B cells (Fig. 6A). Importantly, NAC significantly suppressed G-TPP-induced cell death (Fig. 6B). Various apoptosis assays showed that NAC suppressed G-TPP-induced apoptosis (Fig. 6C and D). NAC also significantly decreased the population of Hep3B cells treated with G-TPP cells that contained elongated mitochondria (Fig. 6E and F). These data indicated that ROS mediates mitochondrial elongation and cell death in G-TPP-treated Hep3B cells. To this end, we examined whether G-TPP reduced the interaction of CDK1 with cyclin B1 and the phosphorylation of Drp1 (pDrp1-Ser616) via ROS. Noticeably, NAC recovered the expression levels of Drp1, CDK1 and p-Drp1 (Ser616) (Fig. 7A); suppressed the G-TPP-induced dissociation of CDK1 from cyclin B1 in Hep3B cells (Fig. 7B); and recovered the recruitment of Drp1 to mitochondria in G-TPP-treated Hep3B cells (Fig. 7C). Flow cytometry revealed that NAC suppressed G-TPP-induced G2-M arrest (Fig. 7D). These results indicate that ROS played a pivotal role in mitochondrial elongation, which is mediated 
A

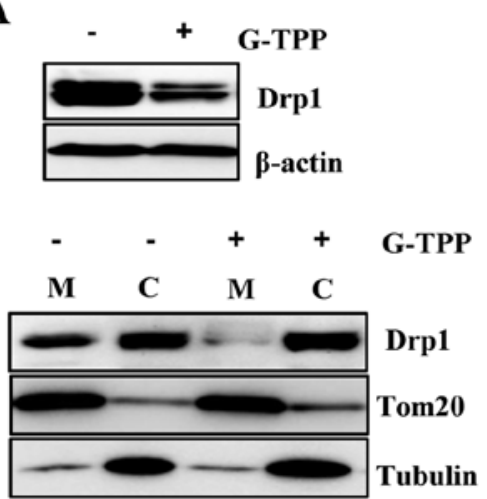

B
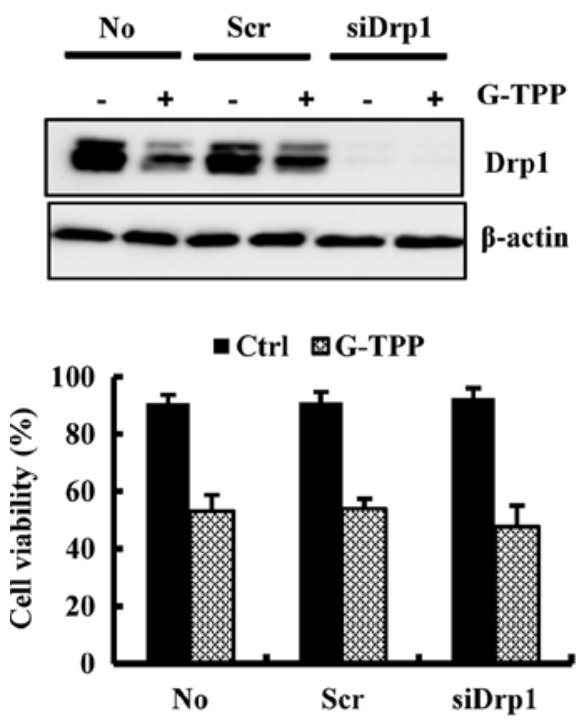

C

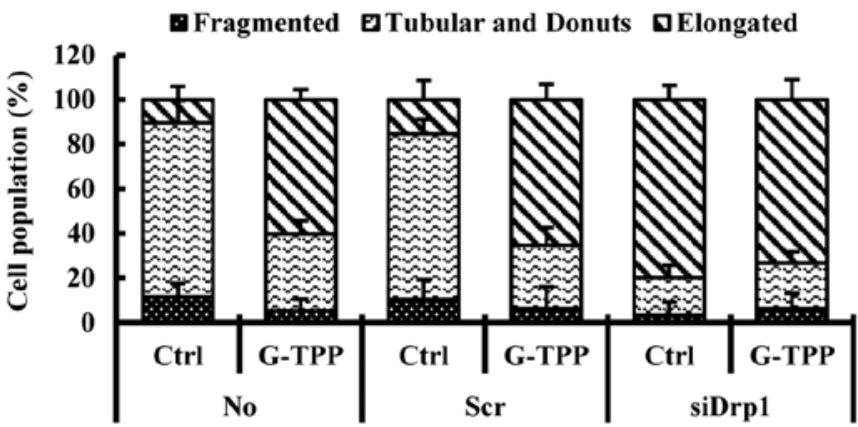

Mitochondrial length ; Fragmented $<2 \mu \mathrm{m}<$ Tubular $<5 \mu \mathrm{m}<$ Elongated
- G-TPP

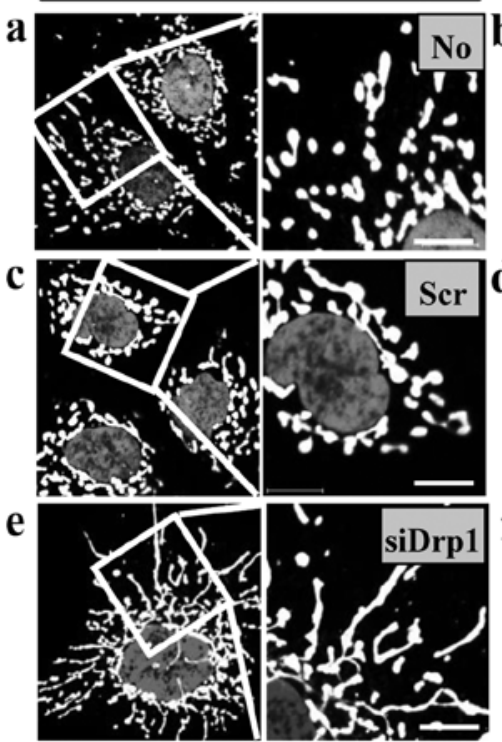

+ G-TPP

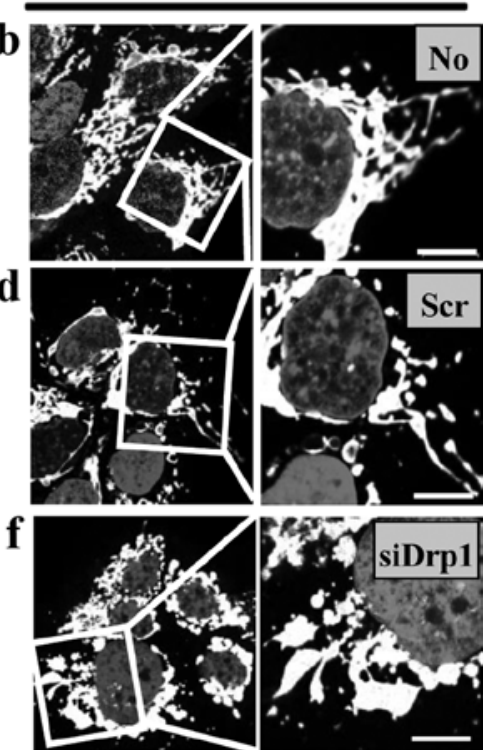

Figure 3. Drp1 is involved in G-TPP-induced mitochondrial elongation. M; mitochondria, C; cytosol. (A) Western blot analyses showing that G-TPP decreased the expression level of Drp1 (upper panel). $\beta$-actin served as a loading control. Western blot analyses showing that G-TPP decreased the amount of Drp1 in mitochondria (lower panel). Tom 20 and tubulin served as mitochondrial and cytosolic markers, respectively. (B) Western blot analyses showing that siDrp1 efficiently depleted Drp1 in Hep3B cells (upper panel). $\beta$-actin served as a loading control. Viability assay showing that siDrp1 did not alter the viability of Hep3B cells treated with G-TPP (lower panel). (C) Quantification assay showing the percentage of cells containing fragmented (dotted), become tubular and donut-shaped (short line) or elongated (striped) mitochondria. siDrpl significantly increased the population of cells with elongated mitochondria in G-TPP-treated and untreated Hep3B compared with scrambled siRNA $(\mathrm{p}<0.05)$. The graphs show the averages of three independent experiments, with 100 cells scored per experiment. (D) Confocal microscopy showing mitochondrial morphology. To visualize the mitochondria and nuclei, cells were stained with Tom 20 (white) and Hoechst 33342 (gray). Scale bar, $10 \mu \mathrm{m}$. See the legend in Fig. 1 for other definitions.

by the reduced association of CDK1 with cyclin $\mathrm{B} 1$ and decreased Drp1 phosphorylation at Ser616 in G-TPP-treated Hep3B cells.

\section{Discussion}

Previous studies reported that the targeted inhibition of mitochondrial Hsp90 using G-TPP induces cell death via ER- and calcium-mediated stress in various cancercells $(21,22)$. Because apoptosis-inducing agents generally induce mitochondrial fragmentation (13-16), we first predicted that G-TPP would induce mitochondrial fragmentation in Hep3B cells. However, we observed that G-TPP induces mitochondrial elongation in Hep3B cells. Mitochondrial fragmentation by apoptotic stimuli depends on the regulation of the mitochondrial fusion-fission balance, which is primarily mediated by the mitochondrial fission machinery $(15,23)$. However, some studies showed that apoptosis-inducing agents could result in mitochondrial elon- gation. A previous study reported that HDAC inhibitors, which induce apoptosis, caused mitochondrial elongation in various cells in addition to inducing apoptosis (24). Our data suggest that G-TPP induces mitochondrial elongation by reducing and inactivating the mitochondrial fission-regulating protein Drp1 and increasing the ROS level.

Because cellular homeostasis is tightly linked to mitochondrial function, the cell must eliminate mitochondria damaged by various stimuli, such as anticancer agents, oxidative stress and starvation. Dysfunctional mitochondria are eliminated via 'mitophagy', a process by which cells selectively remove depolarized mitochondria (25). Parkin, an E3 ligase that was originally discovered as mutated in monogenic forms of Parkinson's disease, has been shown to selectively recognize and eliminate damaged mitochondria (26).

Mitophagy and mitochondrial dynamics are closely correlated (11,27-30). Several previous studies demonstrated that mitochondrial fission is linked to the function of parkin, 


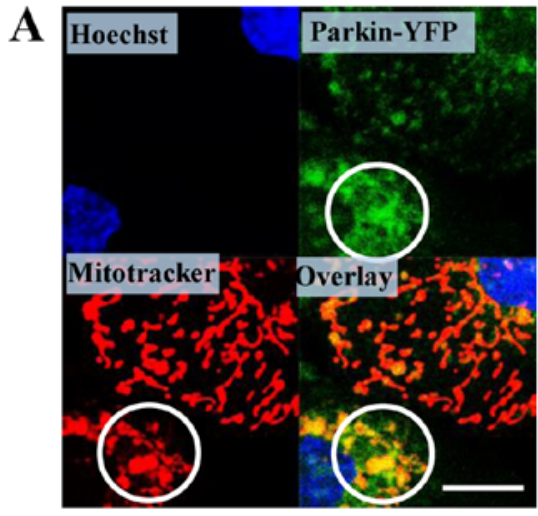

Ctrl

B
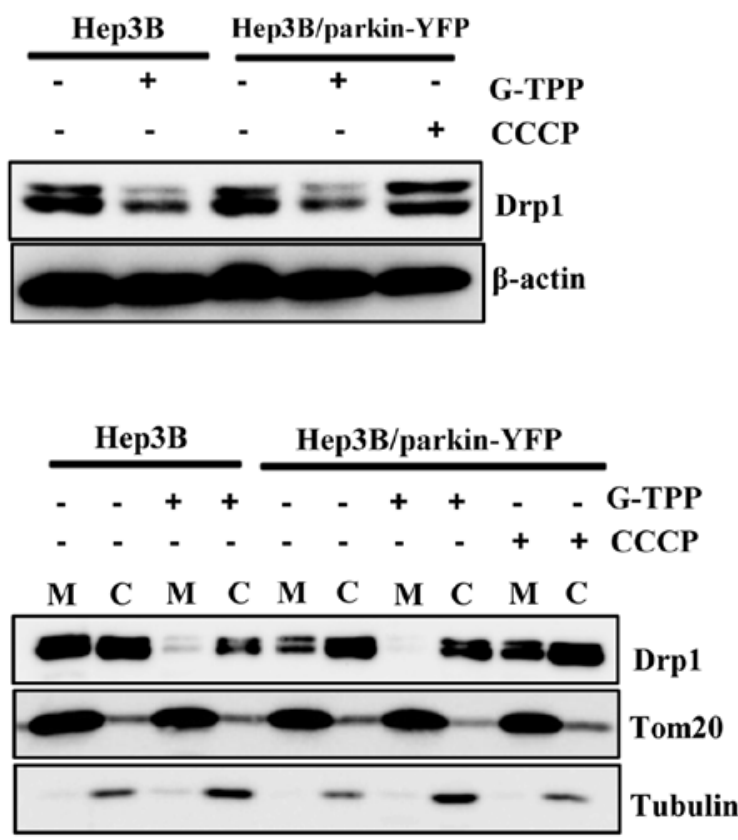

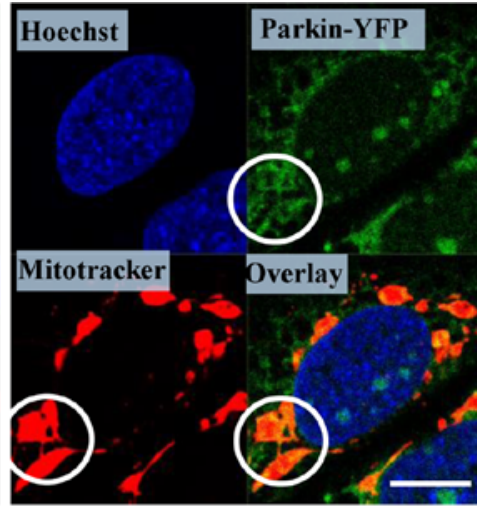

G-TPP

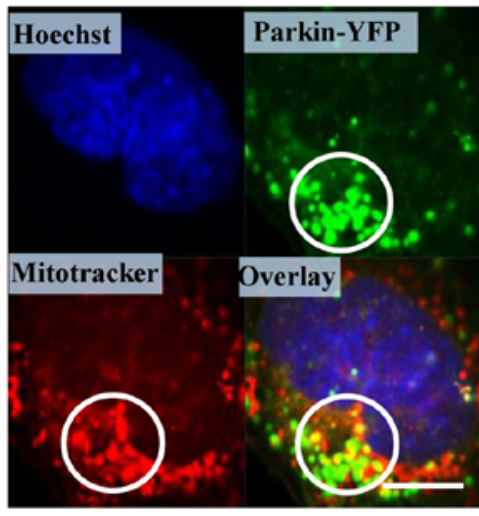

CCCP

C

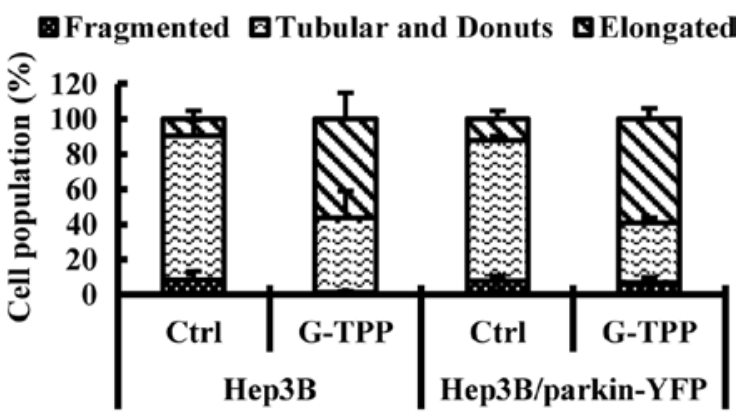

Mitochondrial length ;

Fragmented $<2 \mu \mathrm{m}<$ Tubular $<5 \mu \mathrm{m}<$ Elongated

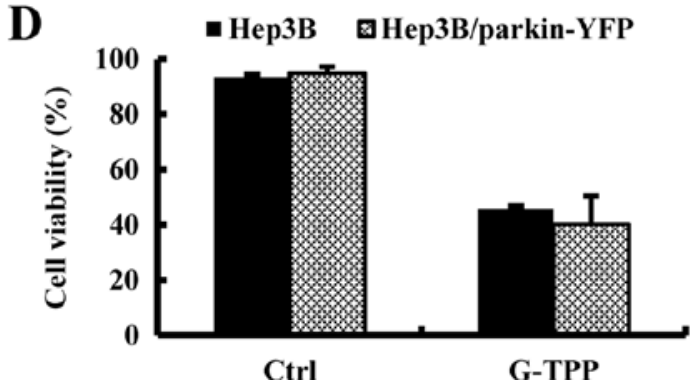

Figure 4. G-TPP suppressed the translocation of Drp1 into mitochondria in YFP-parkin overexpressing Hep3B cells. Cells were treated with $10 \mu \mathrm{M}$ CCCP for $48 \mathrm{~h}$. (A) Confocal microscopy showing that parkin (green) translocated into mitochondria in parkin-overexpressing Hep3B cells irrespective of G-TPP or CCCP treatment. To observe the mitochondria and nuclei, cells were stained with MitoTracker CMXRos (red) and Hoechst 33342 (blue). Scale bar, $10 \mu \mathrm{m}$. (B) Western blot assay showing that G-TPP decreased not only the expression level of Drp1 (upper panel) but the translocation of Drp1 to mitochondria (lower panel) in Hep3B cells irrespective of parkin overexpression. $\beta$-actin served as a loading control. Tom 20 and tubulin served as mitochondrial and cytosolic markers, respectively. (C) Quantification assay indicated that the overexpression of parkin did not affect G-TPP-induced mitochondrial elongation. Quantification assay showing the percentage of cells having fragmented (dotted), tubular and donut-shaped (short line) or elongated (striped) mitochondria. The graphs are averages of three independent experiments, with 100 cells scored per experiment. (D) Viability assay showing that parkin overexpression did not affect G-TPP-induced cell death. See the legend in Fig. 1 for other definitions.
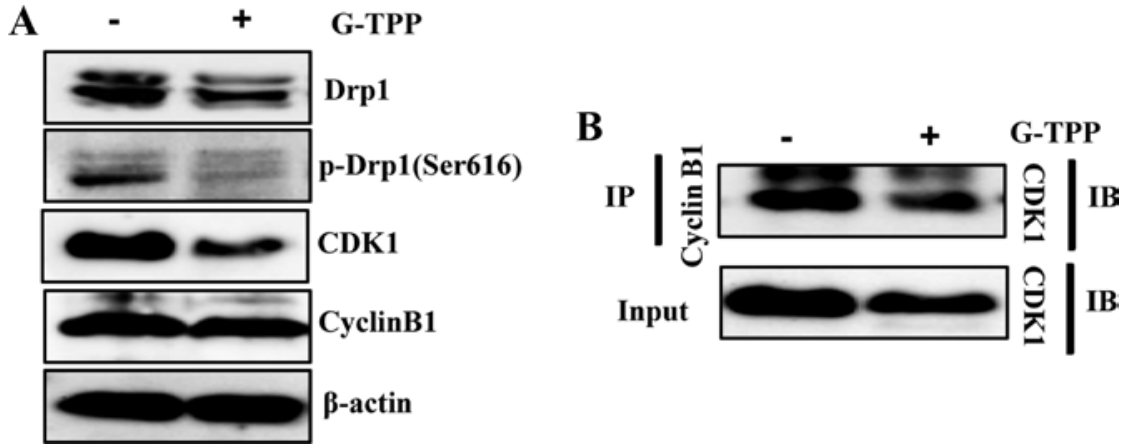

Figure 5. G-TPP reduced the interaction of CDK1 with cyclin B1 and the phosphorylation of Drp1 (Ser616). CDK1; cyclin-dependent kinase 1. (A) Western blot analyses showing that G-TPP markedly decreased the expression level of CDK1 and the phosphorylation of Drp1 (Ser616). $\beta$-actin served as a loading control. (B) Co-immunoprecipitation assay showing that G-TPP decreased CDK1 interaction with cyclin B1. 
A

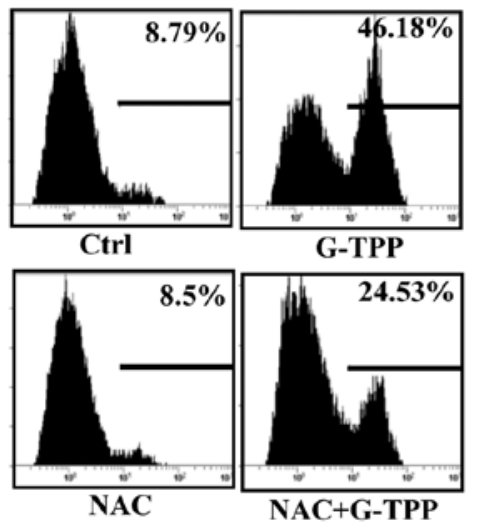

D
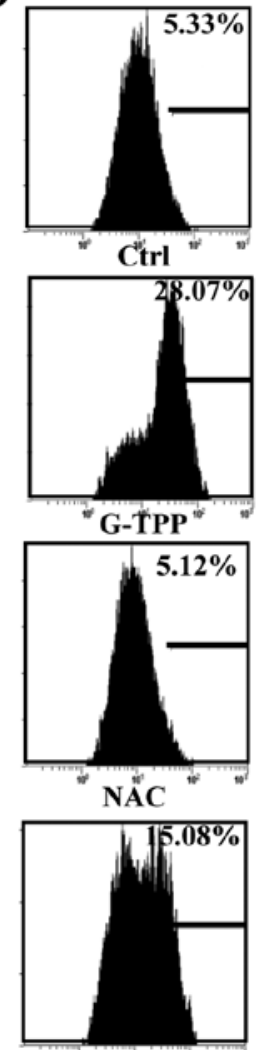

NAC+G-TPP"

JC-1 Green
B

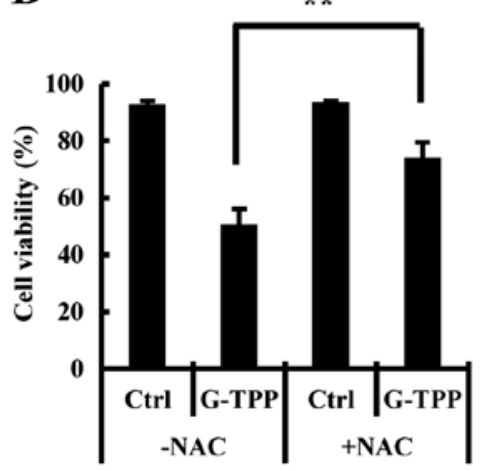

C

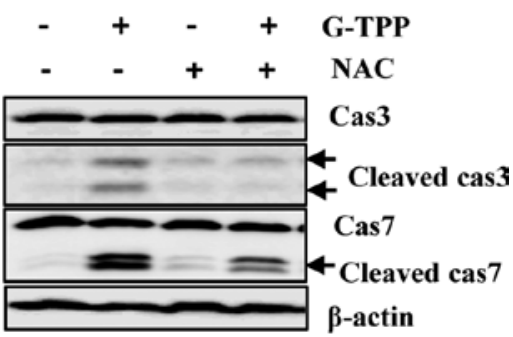

$\mathbf{E}$

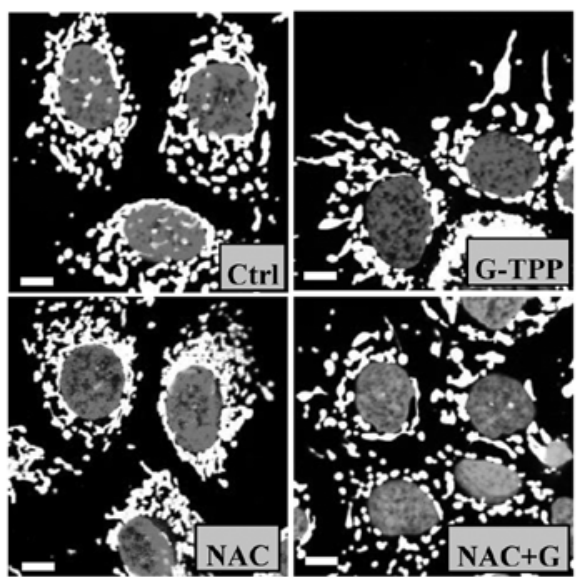

F

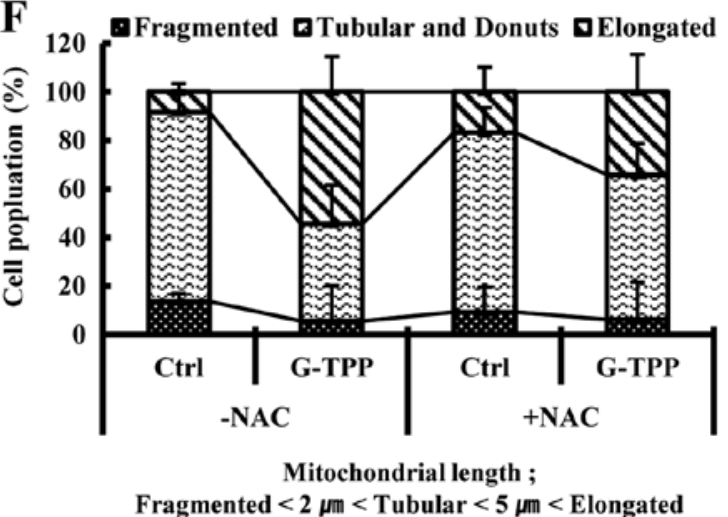

Figure 6. ROS mediated G-TPP-induced cell death and mitochondrial elongation in Hep3B cells. G; G-TPP. Cells were pretreated with NAC (3 mM) for $3 \mathrm{~h}$ and further exposed to $40 \mu \mathrm{M}$ G-TPP for $48 \mathrm{~h}$. (A) Flow cytometry using MitoSOX showing that NAC remarkably inhibited the ROS induced by G-TPP treatment in Hep3B cells. (B) Viability assay showing that NAC significantly suppressed G-TPP-induced cell death. ${ }^{* *}$ p $<0.01$. (C) Western blot analyses showing that NAC prevented the G-TPP-induced activation of caspase-3 and -7. $\beta$-actin served as a loading control. (D) Flow cytometry using JC-1 showing that NAC pretreatment suppressed the G-TPP-induced reduction of the MMP (left panel) and flow cytometry using Annexin V-FITC/PI double staining (right panel) showing that NAC inhibited G-TPP-induced cell death. (E and F) NAC suppressed G-TPP-induced mitochondrial elongation. (E) Confocal microscopy showing mitochondrial morphology. To visualize the mitochondria and nuclei, cells were stained with Tom20 (white) and Hoechst 33342 (gray). Scale bar, $10 \mu \mathrm{m}$. (F) Quantification assay showing the percentage of cells containing fragmented (dotted), tubular and donut-shaped (short line) or elongated (striped) mitochondria. The graphs are averages of three independent experiments, with 100 cells scored per experiment. See the legend in Fig. 1 for other definitions.

and parkin recruitment to mitochondria may be a consequence of depolarization-induced fragmentation $(31,32)$. Whereas staurosporine induced mitochondrial fragmentation and mitophagy in HeLa cells, Drp1 overexpression impaired mitophagy and mitochondrial fission, indicating that mitochondrial fission is required for mitophagy (29). However, a previous study showed that excessive mitochondrial fragmentation alone is insufficient to recruit parkin (26). Mitophagy can be induced independent of parkin (33). While most recent studies have focused on parkin/PINK1-dependent mitophagy, some have examined the parkin/PINK1independent mechanisms of mitophagy (34). The present study suggests that G-TPP induces mitochondrial elongation in Hep3B cells by inhibiting the mitochondrial translocation 

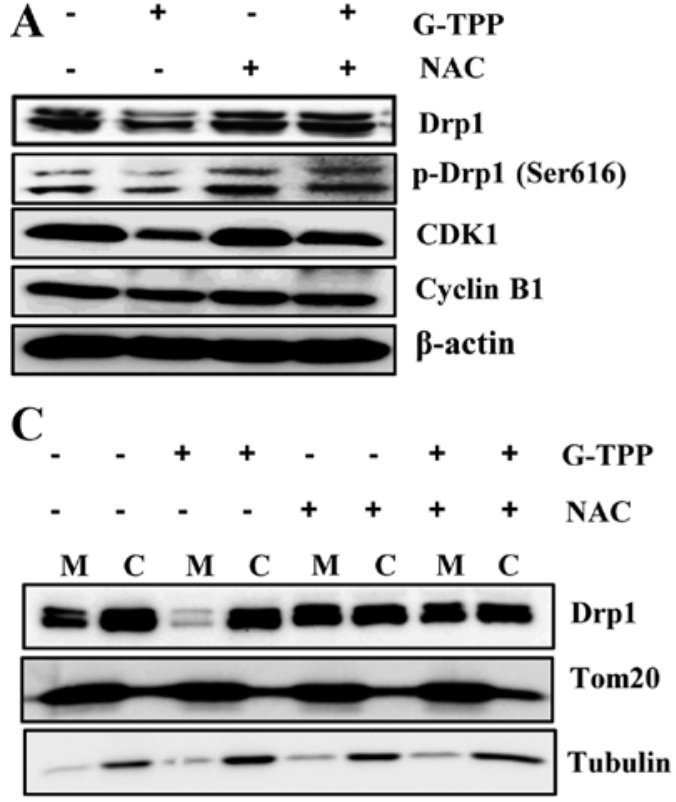

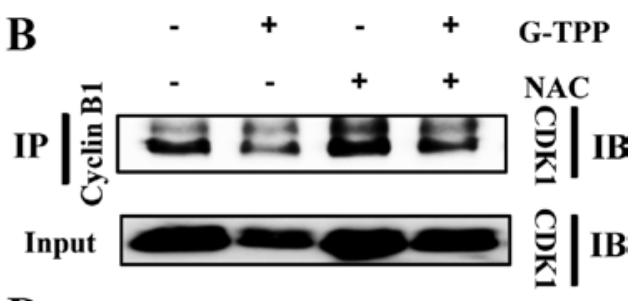

D

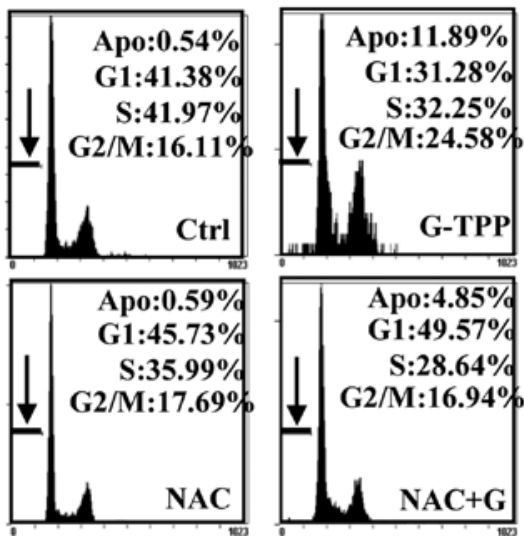

Figure 7. ROS mediated the reduced interaction between CDK1 and cyclin B1 and the inhibition of Drp1 phosphorylation (Ser616). (A) Western blot analyses showing that NAC recovered the expression levels of Drp1, CDK1 and p-Drp1 (Ser616). $\beta$-actin served as a loading control. (B) Co-immunoprecipitation assay showing that NAC suppressed the G-TPP-induced dissociation of CDK1 from cyclin B1 in Hep3B cells. (C) Western blot analyses showing that NAC recovered the recruitment of Drp1 to mitochondria in G-TPP-treated Hep3B cells. Tom20 and tubulin are mitochondrial and cytosolic markers, respectively. (D) Representative histograms indicating that NAC suppressed G-TPP-induced G2-M arrest in Hep3B cells. Apo, the percentage of the population undergoing apoptosis (arrows).

of Drp1, even in parkin-overexpressing cells, which may impair mitophagy. We assume that the induction of apoptosis in G-TPP-treated Hep3B cells is at least partly due to the inefficient removal of dysfunctional mitochondria.

The equal distribution of mitochondria between daughter cells during mitosis is important. Various studies reported that mitochondrial dynamics are integrated with cell cycle progression (35-37). Mitochondrial hyperfusion is known to promote a defect in cell cycle progression characterized by an inability of cells to exit the G2 phase (37). In eukaryotic cells, several cyclin family members regulate the progression from the $\mathrm{G} 2$ to the $\mathrm{M}$ phase. Cyclin B1, together with CDK1, promotes the G2/M transition (38). In particular, loss of Drp1 induced G2 phase arrest (37). The activation of CDK1 and cyclin B1 is a key factor for the G2/M phase transition (36). CDK1-cyclin B1 phosphorylates Drp1 at Ser616 and activates the mitochondrial fission machinery (39-41). This study suggests that G-TPP induces G2 phase arrest via the dissociation of the CDK1-cyclin B1 complex and inhibits Drp1 translocation to the mitochondria.

ROS regulate a wide range of biological processes, including oxygen sensing, immune responses, cell proliferation and differentiation $(42,43)$. The direct or indirect accumulation of ROS lead to apoptosis (44). ROS are primarily produced in mitochondria (45) and mediate mitochondrial dynamics. Numerous studies demonstrated that increased ROS levels mediate mitochondrial fission $(46,47)$. ROS also mediate mitochondrial elongation. A previous study showed that oxidative stress converted elongated tubules into large spheres in fibroblasts (48). Another study reported that the mitochondria of hypoxia-induced chemotherapy-resistant cells undergo a HIF-1-dependent and mitofusin-1-mediated changes in morphology from a tubular network to an enlarged phenotype (49). Moreover, ROS mediate the formation of elongated mitochondria during cellular senescence (50). Our study showed that G-TPP induced mitochondrial elongation in Hep3B cells by increasing the ROS level.

In conclusion, our results indicated that G-TPP induces cell death and causes Drp1-inhibited mitochondrial elongation in Hep3B cells by increasing the ROS level.

\section{Acknowledgements}

This study was supported by a National Research Foundation of Korea (NRF) grant funded by the Korean government (MSIP) (no. 2015 008728).

\section{References}

1. Campello S, Strappazzon F and Cecconi F: Mitochondrial dismissal in mammals, from protein degradation to mitophagy. Biochim Biophys Acta 1837: 451-460, 2014.

2. Picard M, Shirihai OS, Gentil BJ and Burelle Y: Mitochondrial morphology transitions and functions: Implications for retrograde signaling? Am J Physiol Regul Integr Comp Physiol 304: R393-R406, 2013.

3. Harbauer AB, Zahedi RP, Sickmann A, Pfanner N and Meisinger C: The protein import machinery of mitochondria - a regulatory hub in metabolism, stress, and disease. Cell Metab 19: 357-372, 2014.

4. Karbowski M and Youle RJ: Dynamics of mitochondrial morphology in healthy cells and during apoptosis. Cell Death Differ 10: 870-880, 2003

5. Cerveny KL, Tamura Y, Zhang Z, Jensen RE and Sesaki H: Regulation of mitochondrial fusion and division. Trends Cell Biol 17: 563-569, 2007.

6. Benard G and Karbowski M: Mitochondrial fusion and division: Regulation and role in cell viability. Semin Cell Dev Biol 20: $365-374,2009$. 
7. Ishihara N, Fujita $\mathrm{Y}, \mathrm{Oka} \mathrm{T}$ and Mihara K: Regulation of mitochondrial morphology through proteolytic cleavage of OPA1. EMBO J 25: 2966-2977, 2006.

8. Smirnova E, Griparic L, Shurland DL and van der Bliek AM: Dynamin-related protein Drp1 is required for mitochondrial division in mammalian cells. Mol Biol Cell 12: 2245-2256, 2001.

9. Landes T and Martinou JC: Mitochondrial outer membrane permeabilization during apoptosis: The role of mitochondrial fission. Biochim Biophys Acta 1813: 540-545, 2011.

10. Otera H, Wang C, Cleland MM, Setoguchi K, Yokota S, Youle RJ and Mihara K: Mff is an essential factor for mitochondrial recruitment of Drp1 during mitochondrial fission in mammalian cells. J Cell Biol 191: 1141-1158, 2010.

11. Twig G, Elorza A, Molina AJ, Mohamed H, Wikstrom JD, Walzer G, Stiles L, Haigh SE, Katz S, Las G, et al: Fission and selective fusion govern mitochondrial segregation and elimination by autophagy. EMBO J 27: 433-446, 2008.

12. Eisenberg-Lerner A, Bialik S, Simon HU and Kimchi A: Life and death partners: Apoptosis, autophagy and the cross-talk between them. Cell Death Differ 16: 966-975, 2009.

13. Wang IH, Chen HY, Wang YH, Chang KW, Chen YC and Chang CR: Resveratrol modulates mitochondria dynamics in replicative senescent yeast cells. PLoS One 9: e104345, 2014.

14. Pinton P, Ferrari D, Rapizzi E, Di Virgilio F, Pozzan T and Rizzuto R: The $\mathrm{Ca}^{2+}$ concentration of the endoplasmic reticulum is a key determinant of ceramide-induced apoptosis: Significance for the molecular mechanism of Bcl-2 action. EMBO J 20: 2690-2701, 2001.

15. Breckenridge DG, Stojanovic M, Marcellus RC and Shore GC: Caspase cleavage product of BAP31 induces mitochondrial fission through endoplasmic reticulum calcium signals, enhancing cytochrome $\mathrm{c}$ release to the cytosol. J Cell Biol 160 1115-1127, 2003

16. Bai X, Yan Y, Canfield S, Muravyeva MY, Kikuchi C, Zaja I, Corbett JA and Bosnjak ZJ: Ketamine enhances human neural stem cell proliferation and induces neuronal apoptosis via reactive oxygen species-mediated mitochondrial pathway. Anesth Analg 116: 869-880, 2013.

17. Atay C, Ugurlu S and Ozören N: Shock the heat shock network. J Clin Invest 119: 445-448, 2009.

18. Kang BH, Plescia J, Song HY, Meli M, Colombo G, Beebe K, Scroggins B, Neckers L and Altieri DC: Combinatorial drug design targeting multiple cancer signaling networks controlled by mitochondrial Hsp90. J Clin Invest 119: 454-464, 2009.

19. Mayer MP, Prodromou C and Frydman J: The Hsp90 mosaic: A picture emerges. Nat Struct Mol Biol 16: 2-6, 2009.

20. Yamano $\mathrm{K}$ and Youle RJ: Coupling mitochondrial and cell division. Nat Cell Biol 13: 1026-1027, 2011

21. Park HK, Lee JE, Lim J and Kang BH: Mitochondrial Hsp90s suppress calcium-mediated stress signals propagating from mitochondria to the ER in cancer cells. Mol Cancer 13: 148, 2014. doi: 10.1186/1476-4598-13-148.

22. Siegelin MD, Dohi T, Raskett CM, Orlowski GM, Powers CM, Gilbert CA, Ross AH, Plescia J and Altieri DC: Exploiting the mitochondrial unfolded protein response for cancer therapy in mice and human cells. J Clin Invest 121: 1349-1360, 2011.

23. Frank S, Gaume B, Bergmann-Leitner ES, Leitner WW, Robert EG, Catez F, Smith CL and Youle RJ: The role of dynamin-related protein 1 , a mediator of mitochondrial fission, in apoptosis. Dev Cell 1: 515-525, 2001.

24. Lee JS, Yoon YG, Yoo SH, Jeong NY, Jeong SH, Lee SY, Jung DI, Jeong SY and Yoo YH: Histone deacetylase inhibitors induce mitochondrial elongation. J Cell Physiol 227: 2856-2869, 2012.

25. Abeliovich H: Mitophagy: The life-or-death dichotomy includes yeast. Autophagy 3: 275-277, 2007.

26. Narendra D, Tanaka A, Suen DF and Youle RJ: Parkin is recruited selectively to impaired mitochondria and promotes their autophagy. J Cell Biol 183: 795-803, 2008.

27. Twig G and Shirihai OS: The interplay between mitochondrial dynamics and mitophagy. Antioxid Redox Signal 14: 1939-1951, 2011.

28. Ni HM, Williams JA and Ding WX: Mitochondrial dynamics and mitochondrial quality control. Redox Biol 4: 6-13, 2015.

29. Arnoult D, Rismanchi N, Grodet A, Roberts RG, Seeburg DP, Estaquier J, Sheng M and Blackstone C: Bax/Bak-dependent release of DDP/TIMM8a promotes Drp1-mediated mitochondrial fission and mitoptosis during programmed cell death. Curr Biol 15: 2112-2118, 2005
30. Bernhardt D, Müller M, Reichert AS and Osiewacz HD Simultaneous impairment of mitochondrial fission and fusion reduces mitophagy and shortens replicative lifespan. Sci Rep 5: $7885,2015$.

31. Deng H, Dodson MW, Huang H and Guo M: The Parkinson's disease genes pink1 and parkin promote mitochondrial fission and/or inhibit fusion in Drosophila. Proc Natl Acad Sci USA 105: 14503-14508, 2008.

32. Poole AC, Thomas RE, Andrews LA, McBride HM, Whitworth AJ and Pallanck LJ: The PINK1/Parkin pathway regulates mitochondrial morphology. Proc Natl Acad Sci USA 105: 1638-1643, 2008

33. Kageyama Y, Hoshijima M, Seo K, Bedja D, Sysa-Shah P, Andrabi SA, Chen W, Höke A, Dawson VL, Dawson TM, et al: Parkin-independent mitophagy requires Drp1 and maintains the integrity of mammalian heart and brain. EMBO J 33: 2798-2813, 2014.

34. Hirota Y, Kang D and Kanki T: The physiological role of mitophagy: New insights into phosphorylation events. Int J Cell Biol 2012: 354914, 2012

35. Mitra K, Wunder C, Roysam B, Lin G and Lippincott-Schwartz J: A hyperfused mitochondrial state achieved at G1-S regulates cyclin E buildup and entry into S phase. Proc Natl Acad Sci USA 106: 11960-11965, 2009

36. Qian W, Choi S, Gibson GA, Watkins SC, Bakkenist CJ and Van Houten B: Mitochondrial hyperfusion induced by loss of the fission protein Drp1 causes ATM-dependent G2/M arrest and aneuploidy through DNA replication stress. J Cell Sci 125: $5745-5757,2012$.

37. Westrate LM, Sayfie AD, Burgenske DM and MacKeigan JP: Persistent mitochondrial hyperfusion promotes G2/M accumulation and caspase-dependent cell death. PLoS One 9: e91911, 2014.

38. Huang WW, Ko SW, Tsai HY, Chung JG, Chiang JH, Chen KT, Chen YC, Chen HY, Chen YF and Yang JS: Cantharidin induces G2/M phase arrest and apoptosis in human colorectal cancer colo 205 cells through inhibition of CDK1 activity and caspasedependent signaling pathways. Int J Oncol 38: 1067-1073, 2011.

39. Cribbs JT and Strack S: Reversible phosphorylation of Drp1 by cyclic AMP-dependent protein kinase and calcineurin regulates mitochondrial fission and cell death. EMBO Rep 8: 939-944, 2007.

40. Chang CR and Blackstone C: Cyclic AMP-dependent protein kinase phosphorylation of Drp1 regulates its GTPase activity and mitochondrial morphology. J Biol Chem 282: 21583-21587, 2007.

41. Taguchi N, Ishihara N, Jofuku A, Oka T and Mihara K: Mitotic phosphorylation of dynamin-related GTPase Drp1 participates in mitochondrial fission. J Biol Chem 282: 11521-11529, 2007.

42. Yang Y, Bazhin AV, Werner J and Karakhanova S: Reactive oxygen species in the immune system. Int Rev Immunol 32: 249-270, 2013.

43. Matsuzawa A and Ichijo H: Redox control of cell fate by MAP kinase: Physiological roles of ASK1-MAP kinase pathway in stress signaling. Biochim Biophys Acta 1780: 1325-1336, 2008.

44. Zhang Y, Du Y, Le W, Wang K, Kieffer N and Zhang J: Redox control of the survival of healthy and diseased cells. Antioxid Redox Signal 15: 2867-2908, 2011.

45. Chandel NS: Mitochondria as signaling organelles. BMC Biol 12: 34, 2014.

46. Yu T, Robotham JL and Yoon Y: Increased production of reactive oxygen species in hyperglycemic conditions requires dynamic change of mitochondrial morphology. Proc Natl Acad Sci USA 103: 2653-2658, 2006

47. Yu T, Sheu SS, Robotham JL and Yoon Y: Mitochondrial fission mediates high glucose-induced cell death through elevated production of reactive oxygen species. Cardiovasc Res 79: 341-351, 2008.

48. Kageyama Y, Zhang Z, Roda R, Fukaya M, Wakabayashi J, Wakabayashi N, Kensler TW, Reddy PH, Iijima M and Sesaki H: Mitochondrial division ensures the survival of postmitotic neurons by suppressing oxidative damage. J Cell Biol 197: 535-551, 2012.

49. Chiche J, Rouleau M, Gounon P, Brahimi-Horn MC, Pouysségur J and Mazure NM: Hypoxic enlarged mitochondria protect cancer cells from apoptotic stimuli. J Cell Physiol 222: 648-657, 2010.

50. Yoon YS, Yoon DS, Lim IK, Yoon SH, Chung HY, Rojo M, Malka F, Jou MJ, Martinou JC and Yoon G: Formation of elongated giant mitochondria in DFO-induced cellular senescence: Involvement of enhanced fusion process through modulation of Fis1. J Cell Physiol 209: 468-480, 2006. 